\title{
The Shearing Test Instrument Design based on Soliderworks
}

\author{
Huifen Lai \\ College of Mechanical and Electrical Engineering, China jiliang University, Hangzhou, Zhejiang \\ 310018, China \\ huifenlai@163.com
}

Keywords: quality detector, Simulation animation, Shear test instruments.

Abstract. In this paper, a shear force tester is designed and developed. Its mechanical parts of the instrument are calculated and selected to determine the shear tester structure framework. With the SolidWorks software, the standard parts library is established to make the design efficiency greatly and shorten the design cycle of products.

\section{Introduction}

The shearing force testing instrument has the function of measuring the shearing force of the glue on the part surface, mainly through the thimble mechanism to push out the magnet steel product in the fixing and clamping mechanism, and to achieve the measurement of product shearing force in the assembly process curing adhesive glue in the magnetic steel and steel sheet. Its mechanical structure is mainly composed of the thimble positioning mechanism, shear mechanism and clamp mechanism.

Figure 1 shows the schematic diagram of the mechanical structure of the testing instrument.

The foundation

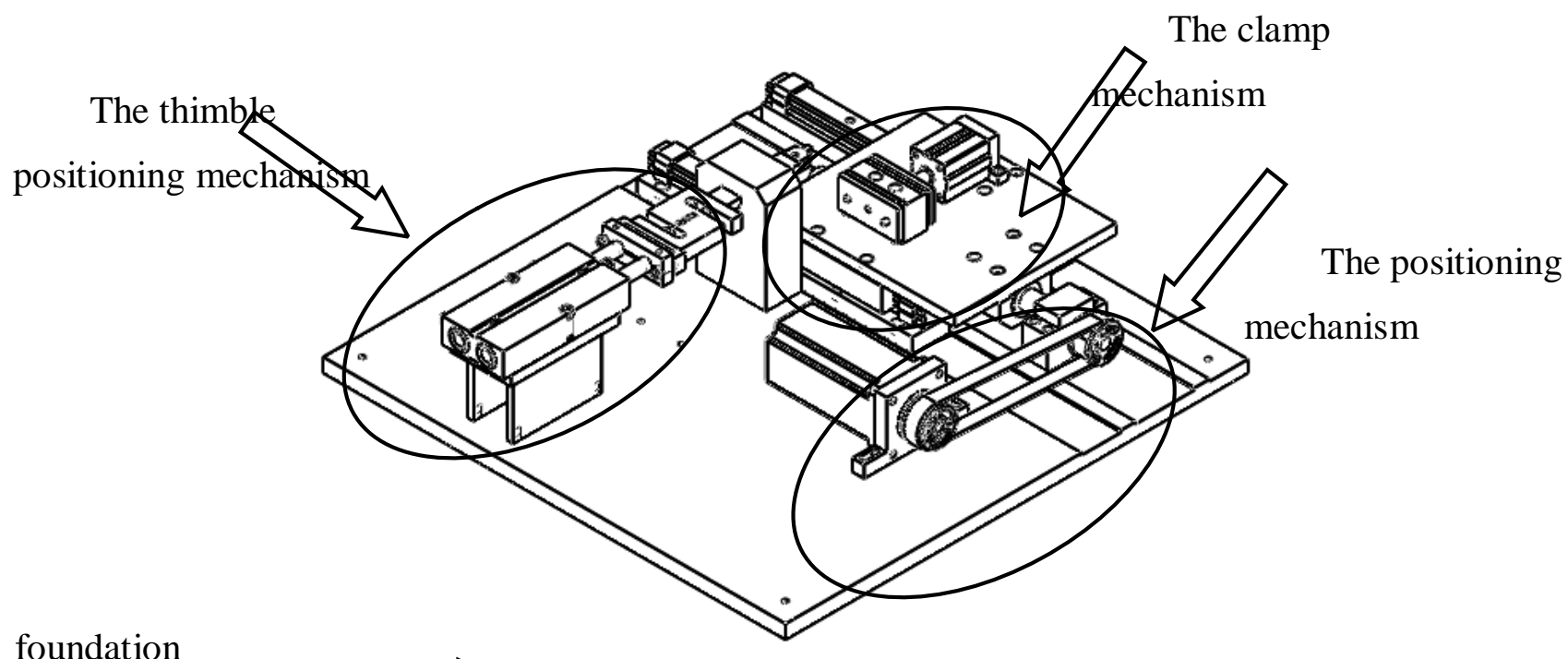

Fig. 1 The main function modules of the testing instrument 


\section{Thimble structure design}

Thimble mechanism is mainly composed of a top needle cylinder, a pressure sensor and a connecting part, provide power by the cylinder. The head of the pressure sensor is connected with a pressure sensor to press the test product, and the data is obtained.

The thimble structure in the shear stress tester as shown in figure 2, Mainly composed of by the biaxial cylinder, the cylinder guide seat, a fixed base, a push rod, thimble and pressure sensors. The cylinder is connected to the pressure sensor through the cylinder connecting block. The thimble powered by biaxial cylinder work drive test objects, the pressure sensor obtain the numerical value reaction of the thimble, this is what we need to test the value of the shear force of the item.

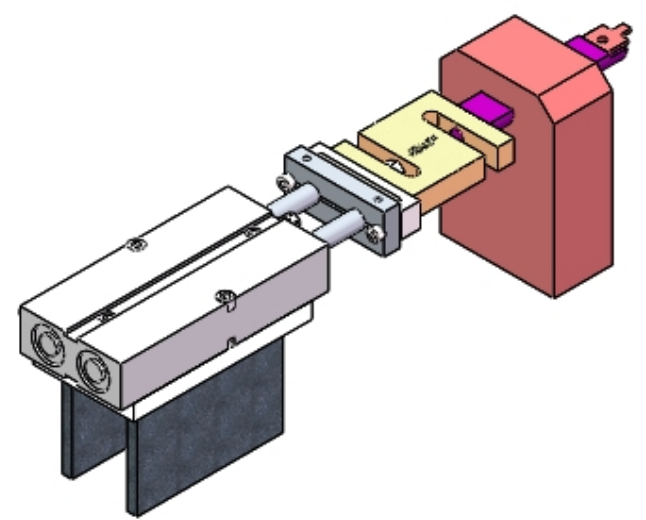

Fig. 2 the thimble structure

\section{Positioning structure design}

The positioning mechanism is composed of a stepping motor, tyre, lead screw and lead rail etc, Mainly by the stepper motor drive the lead screw to location the product $\mathrm{X}$ axis direction.

The main function of positioning structure: the product which is to be detected on the clamping table is shifted to a specific position. The positioning structure is composed of a linear guide rail, a guide rail seat, a sliding block seat, a lead screw, a lead screw fixed seat, a stepping motor and a motor seat.

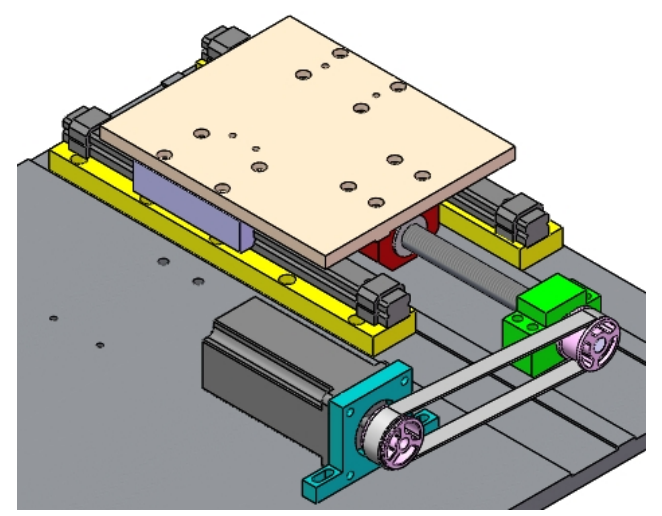

Fig. 3 the positioning structure 


\section{Fixture structure design}

The fixture structure of the shear force tester is to fixed the measured object on the clamping seat to avoid the shift of the object in the experimental process to ensure the accuracy of the experimental data. The fixture mechanism is composed of an air cylinder, a clamping seat, a connecting shaft and a pressing plate. The fixture structure is provided with a reaction force through connecting the shaft with the cylinder to clip the measured product in the reserved slot in the middle of the clamping plate and the clamping seat.

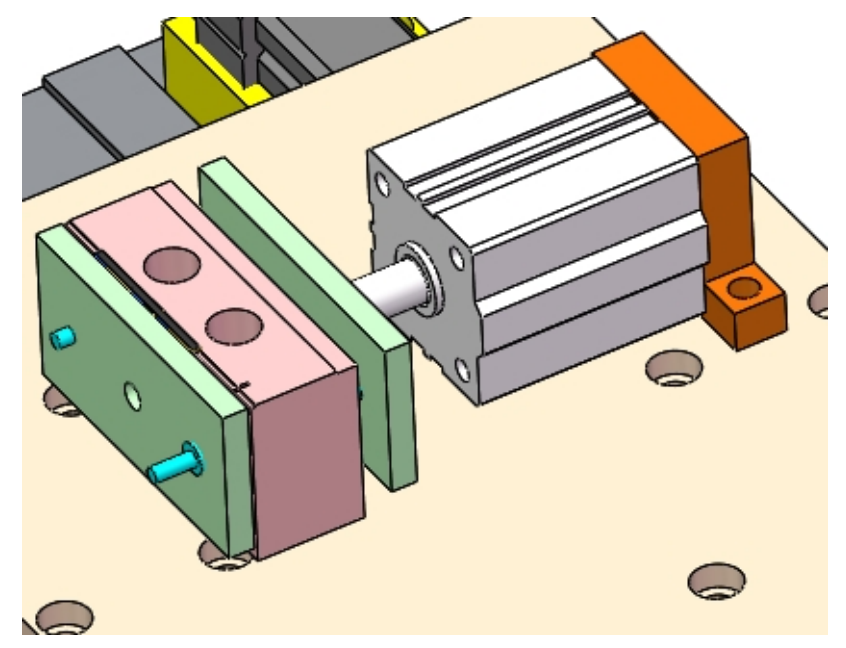

Fig. 4 the fixture structure

\section{Shear force tester assembly}

With the Soliderworks software, the three-dimensional model of shear force testing instrument was established, the model was simulated and assembled, and the process was analyzed through the movement of Soliderworks software, and its motion trajectory was simulated.

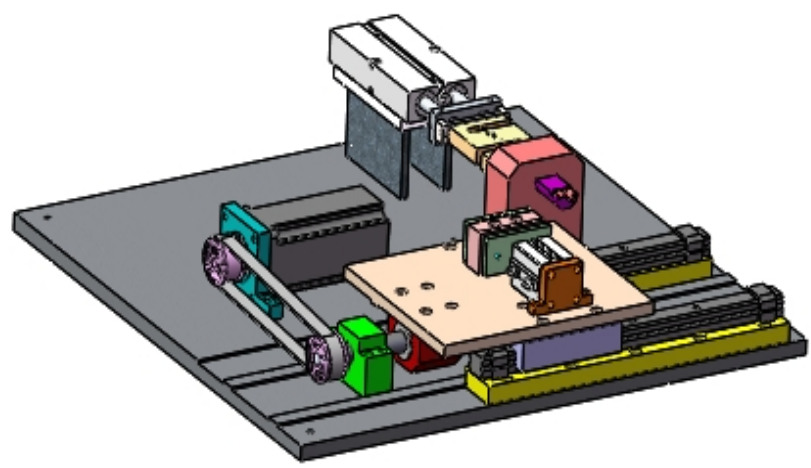

Fig. 5 Shear force tester assembly

\section{Conclusion}

This design analyzes the current situation of the shear force tester in our country, combined with 
the practical application and the existing technical level, to understand the current domestic and international shear testing instrument. According to the existing shear force testing instrument in the market, the main parts and assembly are designed, and the process analysis of the three-dimensional modeling and production of parts is carried out.

By the existing shearing force testing instrument for split, the parts of the analysis and calculation, simulation, and assembly, a practical shear force tester is designed.

\section{References}

[1] Zhu Honglin, The core pulling slider with a thimble mechanism design . Hydraulic and pneumatic, 2010 (4).

[2] Wang Xiongyao, The low speed and high speed cylinder . Chinese Journal of mechanical engineering $2006(9)$.

[3] Wang Shukun, the positioning accuracy of ball screw feed system. Dalian University of Technology, 2010,06.

[4] Zhou Guihua, Li Chuanduo, Sun Songlin, Wang Shulei. Application of hydraulic transmission mechanism in the transformation of metal packaging machine. Machinery Industry Press. 2011,01. 